\title{
A Novel Integration Forecasting Approach for Short-Term Wind Power
}

\author{
Kailin Zhao ${ }^{1,2}$, Lingyun Wang ${ }^{1,2, *}$ and Qiwei Ma ${ }^{1,2}$ \\ ${ }^{1}$ College of Electrical Engineering and New Energy, China Three Gorges University, Yichang, 443002, China \\ ${ }^{2}$ Hubei Provincial Collaborative Innovation Center for New Energy Microgrid, Yichang, 443002, China \\ *Corresponding author
}

\begin{abstract}
As a kind of renewable energy, wind power energy has the characteristics of randomness and intermittence. The integration of wind farm will affect the safe and stable operation of power grid. Therefore, the accuracy of wind power output prediction is very important for power system operation and power quality. In order to improve the accuracy of short-term wind power forecasting, this paper presents a novel integration prediction model base on wind power and wind speed. The combination forecasting model of autoregressive time series and generalized regression neural network is used to forecast the wind power generation directly and forecast the wind power generation indirectly by wind speed. The final prediction model is obtained by combining the two models. The results show that the prediction accuracy of the model is improved effectively.
\end{abstract}

Keywords-wind speed, power forecasting; autoregressive time series; integration forecasting model; generalized regression neural network

\section{INTRODUCTION}

Wind farm is a new kind of low-cost way to generate electricity with great promise. However, the greatest problem of wind farm to the existing power system is intermittent and variable nature of wind power due to the high correlation with stochastic nonstationary of wind speed. For this reason, a reliable and decisive wind speed and wind power forecasting model is a fundamental necessity for mitigating the undesirable results in wind energy conversion systems. In Ref.[1], Cassola uses Kalman filtering to forecast wind energy and combines recursively observations and model forecasts to minimise the corresponding biases. In Ref.[2], in order to overcome the disadvantages in traditional approaches such as computing burden and low accuracy, model structure selection method is used to forecast output power of a wind power plant. In Ref.[3], Shao predicts wind power with wavelet transformation, solving the defects of the lower accuracy and enhance the model robustness.

In this paper, in order to improve the precision of the short term output wind power, an integration forecasting model of autoregressive time series and generalized regression neural network based on wind speed and wind power is proposed. At last, this approach is verified effectively through the actual engineering data.

\section{THE ANALYSIS OF WIND POWER CHARACTERISTICS}

Generally, the relationships between output power of the wind farm and wind speed is nonlinear. The power characteristics of a wind turbine including cut-in, rated output and cut-out speeds. For the power characteristic of a model wind turbine, at very low wind speeds, there is insufficient torque exerted by the wind on the turbine blades to make them rotate. However, as the speed increases, the wind turbine will begin to rotate and generate electrical power. The speed at which the turbine firstly starts to rotate and generate power is called the cut-in speed and is typically between 3 and $4 \mathrm{~m} / \mathrm{s}$. As the wind speed rises above the cut-in speed, the output power level rises rapidly as shown. However, typically somewhere between 12 and $17 \mathrm{~m} / \mathrm{s}$, the power output reaches the limit that the electrical generator is capable of. This limit to the generator output is called the rated power output and the wind speed at which it is reached is called the rated output wind speed. At higher wind speeds, the design of the turbine is arranged to limit the power to a maximum level and there is no further rise in the output power. How this is done varies from design to design but typically with large turbines, it is done by adjusting the blade angles so as to keep the power at the constant level. As the speed increases above the rate output wind speed, the forces on the turbine structure continue to rise and, at some point, there is a risk of damage to the rotor. As a result, a braking system is employed to bring the rotor to a standstill. This is called the cut-out speed and is usually around $25 \mathrm{~m} / \mathrm{s}$.

The prediction of a single model can not meet the requirements of the short term forecasting of wind power, and the combination of multi model will greatly improve the prediction accuracy and improve the impact of wind power on the grid.

\section{A. Autoregressive Time Series}

The time series is one of the main methods for the short-term prediction for the output power of the wind farm. This method is relatively simple, just using the wind speed time series to forecast the output power. When the wind power output is predicted concretely, the time series model is to determine the value of the model order and estimate the model parameters and variance. Generally, Final Predition Error criterion and Akaike Information Criterion information criterion are used to judge and determine the appropriate order of the model ${ }^{[4]}$. 
Assuming that there is a linear relationship between the data Xt of the Autoregressive time series $\left\{X_{t} \mid t=1, \ldots, n\right\}$ and $X_{t-1}$, $X_{t-2}, \ldots, X_{t-n}$. In the case of the known of $X_{t-1}, X_{t-2}, \ldots, X_{t-n}, X_{t}$ and $X_{t-j}(j=n+1, n+2)$ are unrelated. In the Autoregressive time series $\left\{X_{t}\right\}$, the model describing the relationship between different times of the time series $\left\{X_{t}\right\}$ is:

$$
X_{t}=\varphi_{1} X_{t-1}+\varphi_{2} X_{t-2}+\cdots+\varphi_{n-1} X_{t-n+1}+\varphi_{n} X_{t-n}+\varepsilon_{t}
$$

where, $\left\{\varphi_{1}, \varphi_{2}, \cdots, \varphi_{n-1}, \varphi_{n}\right\}$ are the variable coefficients. $\left\{\varepsilon_{t}\right\}$ is a white noise sequence and independent of sequence $\left\{X_{t-1}, X_{t-2}, X_{t-3}, \cdots, X_{t-n}\right\}$.

\section{B. Generalized Regression Neural Network}

The theoretical basis of the GRNN(Generalized Regression Neural Network) is nonlinear regression analysis. Different from the BP(Backpropagation) Neural Network, GRNN is composed by four layers: the input layer, the pattern layer, the summation layer and the output layer. Different from BP Neural Network, GRNN is composed by four layers: the input layer, pattern layer, summation layer and output layer. According to the training process of GRNN, once the training samples are determined, the network structure and the connection weights between the neurons are also determined. The only factor that affects the output of the network is the smoothing parameter $\sigma$.The smaller the smoothing factor is, the stronger the approximation of the network to the sample is, meanwhile, the greater the smoothing factor is, the smoother the approximation of the sample is ${ }^{[5-6]}$.

\section{Combination Model by Forecasting Power Directly (Model} 1)

Under different conditions the calculation precision of each prediction method may be different, against the advantage of the different models, the method of variable coefficients is adopted to construct combination model. This way can further improve the prediction precision.

Under different conditions the calculation precision of each prediction method may be different, against the advantage of the different models, the method of variable coefficients is adopted to construct combination model. This way can further improve the prediction precision. The combination model is set up, such as formula (5), the determination of the weight coefficient of the model has nothing to do with the data sample, and the weight coefficient changes over time.

$$
\hat{P}_{1}=\lambda_{1} P_{1}+\lambda_{2} P_{2}
$$

where, $\hat{P}_{1}$ is the total forecast output power. $P_{1}$ and $P_{2}$ are the output power of AR(Autoregressive time series) and GRNN(Generalized Regression Neural Network) model respectively. $\lambda_{1}, \lambda_{2}$ are the corresponding weight coefficients, and $\lambda_{1}+\lambda_{2}=1$.

Assuming the errors of the two kinds of prediction models are $e_{1}$ and $e_{2}$, the variance of combined prediction error is $\operatorname{var}\left(e_{c}\right)=\sum_{i=1}^{2} \lambda_{i}^{2} \delta_{i}, \delta_{i}$ is the variance of ith prediction method. Under the constraint of $\lambda_{1}+\lambda_{2}=1$, introducing the Lagrange multiplier method to calculate the minimization value of $\operatorname{var}\left(e_{c}\right)$,we can get

$$
\begin{gathered}
\lambda_{i}=\frac{1}{\delta_{i}\left(1 / \delta_{1}+1 / \delta_{2}\right)}, \quad i=1,2 \\
\operatorname{var}\left(e_{c}\right)=\frac{1}{1 / \delta_{1}+1 / \delta_{2}}
\end{gathered}
$$

When the errors of these two kinds of the prediction methods satisfy the zero mean normal distribution, we can estimate from the following formula

$$
\hat{\delta}_{i}=\frac{1}{n} \sum_{t=1}^{n} e_{i t}^{2}, \quad i=1,2
$$

where $n$ is the dimension of historical data, $e_{i t}$ is the error of the ith combination model at time point $t$. It can be concluded that the estimated value of $\lambda_{i}, i=1,2$ is

$$
\hat{\lambda}_{i}=\left(\sum_{t=1}^{n} e_{i t}^{2}\right)^{-1}\left[\sum_{j=1}^{2}\left(\sum_{t=1}^{n} e_{j t}^{2}\right)^{-1}\right]^{-1}
$$

Substituting the estimated value of $\lambda_{i}$ into the above formula, we can get the combined forecasting model is

$$
P_{\sum}=\sum_{i=1}^{2}\left(\left(\sum_{t=1}^{n} e_{i t}^{2}\right)^{-1}\left[\sum_{j=1}^{2}\left(\sum_{t=1}^{n} e_{j t}^{2}\right)^{-1}\right]^{-1} P_{i}\right)
$$

Through the above analysis, it can be proved that the combination forecasting method is better than the single prediction method,so the model is further optimized. 
D. Combination Model by Forecasting Power Based on Wind Velocity (Model 2).

In the case of that the two models of AR(Autoregressive time series) and GRNN(Generalized Regression Neural Network) are used to forecast wind speed respectively, still using the method of combination model, combining the advantages and disadvantages of the two models. Introducing weighting coefficients, the two models will be combined. At this point, the combination model is:

$$
\hat{v}=\lambda_{1} v_{1}+\lambda_{2} v_{2}
$$

where, $\hat{v}$ is the total forecasting wind speed. $v_{1}, v_{2}$ are the calculated speed through the models of AR and GRNN respectively.

Using the similar method of combination forecasting model one, it can be deduced that a new combination forecasting model is

$$
\hat{v}=\sum_{i=1}^{2}\left(\left(\sum_{t=1}^{n} e_{i t}^{2}\right)^{-1}\left[\sum_{j=1}^{2}\left(\sum_{t=1}^{n} e_{j t}^{2}\right)^{-1}\right]^{-1} v_{i}\right)
$$

where $v_{i}$ is the result of the ith iteration. $e_{i t}$ is the prediction error in the $i$-th iteration. $\hat{v}$ is the forecasting wind speed of combination model. According to forecasting wind speed, using the relationship (2) between output power and wind speed, the power $\hat{P}_{2}$ of each time can be calculated. At this point, the error of the combination model will become smaller.

\section{E. Integration model based on wind velocity and output power (Integration Model)}

Considering that the data of the AR(Autoregressive time series) model always changes over time, the value in every moment has randomness. Overall, time series are often present a trend or cyclical changes. So the relative error will be small by using this model. And the network structure of GRNN(Generalized Regression Neural Network) is relatively simple, the calculation of this model is generally global convergence. Considering the features of these two models, a integration model based on the wind velocity and wind power is proposed, combining the Model 1 and the Model 2 mentioned above, the new model is as below:

$$
\hat{P}=\lambda_{1} \hat{P}_{1}+\lambda_{2} \hat{P}_{2}
$$

where, $\hat{P}$ is the total forecasting output power. $\hat{P}_{1}$ is the forecasting power of the combination model $1 . \hat{P}_{2}$ is the forecasting power of the combination model 2. Obviously, the prediction accuracy of the combination model is much higher than the prediction accuracy of a single model.

\section{EXAMPLES VERIFICATION}

In this paper, all the data is selected from a wind farm in Jilin province, China and the types of all the wind turbines in this wind farm are G52/58-850KW produced by Gamesa Company, Spain. The data used for wind power prediction is derived from the actual production data of the wind farm in one month of the year, meanwhile all the data is collected every 15 minutes.

In addition, the wind energy utilization coefficient of the wind turbines is not a constant, it changes with other parameters like wind speed and revolving speed. So the polynomial fitting is used generally to establish the characteristic curve of the wind turbines. According to the actual sample data of wind velocity, with the help of Polyfit fitting function in matlab simulation software, the polynomial equations of wind speed and wind power is established as follow:

$$
P=0.0114 v^{3}-4.2944 v^{2}+149.4164 v-497.9861
$$

Figure 1 shows the relationship between the wind speed and the wind power.

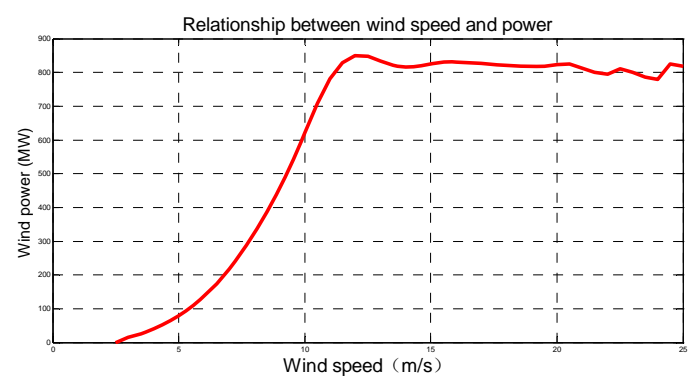

FIGURE I. THE RELATIONSHIP BETWEEN WIND SPEED AND WIND POWER

The specific data of the wind farm will be used to verify the integration model. Based on this model, the updated time-varying weight coefficients of two models should be solved. According to the method proposed in this paper, Figure 2- 3 show the prediction results of the established models.
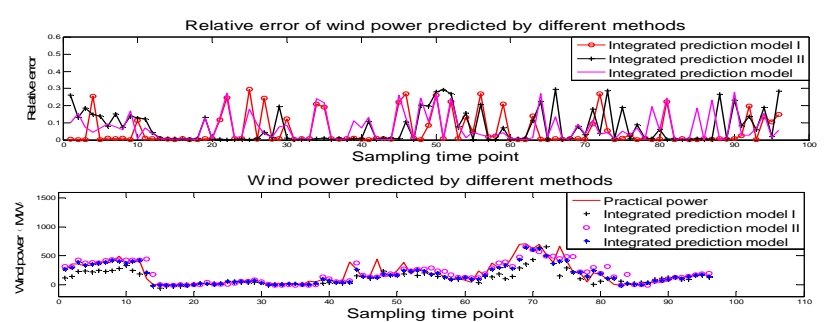

FIGURE II. POWER AND PREDICTION ERROR OF COMBINATION MODELS 


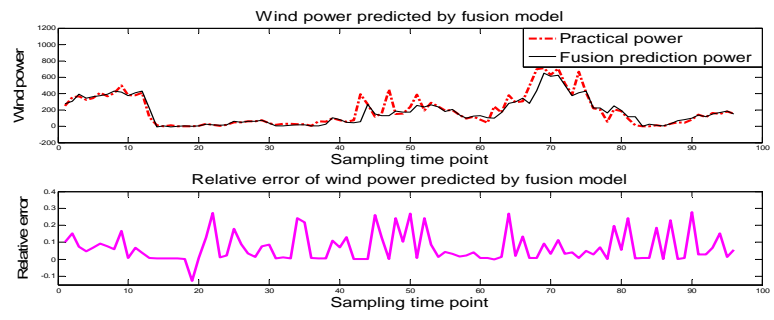

FIGURE III. RESULT OF INTEGRATION MODEL

According to the Figure 3, it can be seen that the prediction value of the integration model is close to the real wind power mostly. In the process of prediction, the error in every moment is the smallest, the total error is $7.16 \%$.

TABLE I. COMPARISON OF PREDICTION SCHEMES FOR DIRECT PREDICTION OF WIND POWER

\begin{tabular}{cc}
\hline Prediction Schemes & Relative Error \\
\hline Prediction model of AR & $28.8 \%$ \\
Prediction model of GRNN & $18.3 \%$ \\
Combination forecasting model & $16.3 \%$ \\
\hline
\end{tabular}

TABLE II. COMPARISON OF PREDICTION SCHEMES FOR WIND POWER BASED ON WIND SPEED FORECASTING

\begin{tabular}{cc}
\hline Prediction Schemes & Relative Error \\
\hline Prediction model of AR & $21.1 \%$ \\
Prediction model of GRNN & $26.4 \%$ \\
Combination forecasting model & $13.8 \%$ \\
\hline
\end{tabular}

\section{TABLE III. COMPARISON OF COMBINED FORECASTING SCHEMES BASED ON WIND SPEED AND WIND POWER}

\begin{tabular}{cc}
\hline Prediction Schemes & Relative Error \\
\hline Prediction model of AR & $16.3 \%$ \\
Prediction model of GRNN & $13.8 \%$ \\
Combination forecasting model & $7.2 \%$ \\
\hline
\end{tabular}

According to the calculated relative error, the relative error of GRNNmodel is smaller than AR model in the model 1. But the relative error of combination model is far less than the two methods, it is $16.32 \%$. In the model 2 , the relative error of the AR model is smaller than the GRNN model, but the combination model is still better than the two methods, its relative error is $13.82 \%$. Finally, combining the model 1 and model 2 to get the ingration model, its relative error is $7.16 \%$. As known from the above analysis, the integration model proposed in this paper is feasible and effective. Not only the meteorological information is used in the prediction, but also the complexity is reduced. So the prediction precision of large wind farm output power is effectively improved.

\section{SUMMARY}

In this paper, a novel integration model has been studied. Considering the main factors in the power prediction, the power prediction and wind speed prediction models are combined together to establish an integration model, which can improve the precision of short term power prediction. According to the simulation verification with the practical production data from the wind farm, the prediction precision of integration model is much higher than the single model.

\section{ACKNOWLEDGEMENTS}

This work is supported by the National Natural Science F-oundation of China (51407104).

\section{REFERENCES}

[1] F. Cassola, M. Burlando. "Wind speed and wind energy forecast through Kalman filtering of numerical weather prediction model output, ” Applied Energy, vol. 99, pp. 154-166, 2012.

[2] H. Wei, H. Shao, X. Deng. "Using a model structure selection technique to forecast short-term wind speed for a wind power plant in North China," Journal of Energy Engineering, vol. 142, 04015005, 2016.

[3] H. Shao, X. Deng, F. Cui. "Short-term wind speed forecasting using the wavelet decomposition and AdaBoost technique in wind farm of East China," IET Generation, Transmission and Distribution, vol. 10, pp. 2585-2592, 2016.

[4] I. Colak, S. Sagiroglu, M. Yesilbudak, E. Kabalci and H.I. Bulbul "Multi-time series and -time scale modeling for wind speed and windpower forecasting - Part II: Medium-term and long-term applications," 4th International Conference on Renewable Energy Research and Applications, pp. 22-25, Palermo, Italy, November 2015.

[5] N. Amjady, F. Keynia, H. Zareipour. "Wind power prediction by a new forecast engine composed of modified hybrid neural network and enhanced particle swarm optimization,” IEEE Trans. on Sustainable Energy, vol. 2, pp. 265-276, 2011.

[6] G. Sideratos, N. Hatziargyriou. "Using radial basis neural networks to estimate wind power production,” IEEE Power Engineering Society General Meeting, PES, June 24, 2007 - June 28, 2007. 